\section{P165 IMPROVING TRANSITION SERVICES IN IRELAND - WHAT ARE THE BARRIERS TO SUCCESS?}

${ }^{1}$ Jayne MacMahon*, ${ }^{2}$ Yvonne Owens, ${ }^{3}$ Naomi Oldenburg, ${ }^{1}$ Orla G Killeen. ${ }^{1}$ National Centre for Paediatric Rheumatology, Our Lady's Children's Hospital Crumlin, Dublin, Ireland; ${ }^{2}$ Royal College of Physicians, Dublin, Ireland; ${ }^{3}$ Beaumont Hospital, Dublin, Ireland

10.1136/archdischild-2019-epa.520

Introduction Transition is an important concept in the management of young people and adolescents (YPA) with long term or chronic illness and refers to the planned move of YPA from their paediatric health care provider across to adult services. The importance of a planned, successful transition has been highlighted in recent years, after multiple studies showed that a poor transition can lead to poor patient adherence, outpatient attendance and overall decline in patient outcomes. In order to develop a more structured transition programme, the key areas of need, from the point of view of YPA and health care workers, should be identified.

Aim To evaluate health care workers perception of the current transition process in Ireland and identify areas of need and concern.

Methods An 18 question survey was developed and distributed amongst health care workers in both the adult and paediatric services via survey monkey. The responses were then collated and analysed using Microsoft Excel.

Results The survey registered 143 responders in total, with an average of 141 responders per question (range 136-143). $78 \%$ worked in a paediatric healthcare, and $57 \%$ of those reported working in a tertiary care setting. 70\% were directly involved in the transfer of YPA from paediatric to adult services. A structured transition programme was felt to be 'very important' by $92 \%$ of responders, with the majority (87\%) suggesting between 12 and 16 years as the most appropriate ages to start this process. Notably, $73 \%$ felt that Irish YPA were inadequately prepared for the move to adult services and highlighted poor access to adult services, lack of resources and lack of communication between paediatric and adult services as the biggest barriers to a successful transition.

Conclusion This study identified some of the main barriers to a successful transition, from the point of view of health care workers. The need for a structured transition programme, along with guidelines and increased resources was particularly highlighted.

\section{P166 TERTIARY IN-REACH CLINIC DOCUMENTATION}

Phillip Ross*, Andrew Thompson. Royal Belfast Hospital for Sick Children, Belfast, UK

\subsection{6/archdischild-2019-epa.521}

Introduction In-reach clinics involve specialist paediatric clinicians who travel to Royal Belfast Hospital for Sick Children (RBHSC) from mainland UK. They undertake joint clinics with RBHSC consultants - providing specialist care for patients closer to home.

The O'Hara enquiry stated record keeping should be subject to rigorous, routine and regular audit. We wanted to review the quality of documentation from our specialist inreach clinics for several reasons. We wanted to ensure it was in keeping with standardised practice to maximise patient safety, quality of care and to support professional best practice.
Methods We reviewed 20 sets of patients notes from five different speciality in-reach clinics. We reviewed the notes looking at 17 different criteria. We based our criteria on the generic medical record keeping standards produced by the Royal College of Physicians. They criteria were as follows:

1. Was a record of clinic appointment made in the notes

2. Which clinician present documented the clinic appointment

3. Was the patients first and last name on each page of record

4. Was the patients identification number present on record

5. Was the date of clinic appointment recorded

6. Was the time of the clinic appointment recorded

7. Was the record signed by clinician making the entry

8. Was the clinician's name legibly printed

9. Was the clinicians general medical council number printed against signature

10. Was it documented which other healthcare professionals where present during clinic appointment

11. Where deletions and alterations countersigned, dated and timed

12. Was the patients medical history recorded

13. Were the examination finding recorded

14. Were the patients investigations recorded

15. Was the patients diagnosis recorded

16. Was the patients management plan recorded

17. Was the patients clinic letter available on Electronic Care Record

Results The healthcare professionals present during the clinic was documented in $35 \%$ of records. The examination findings were documented in $40 \%$ of records. The name of clinician making record was legible in $35 \%$ records. The clinicians General medical council number was documented in $10 \%$ of records.

Conclusion We recognised there were areas for significant improvement on documentation. We are currently in the process of providing a proforma for in-reach clinics. This will be provided to clinicians and available in outpatient clinic rooms at Royal Belfast Hospital for Sick Children. The proforma will provide a layout for the standard of documentation which would be expected for in-reach outpatient clinic appointments.

\section{P167 IMPROVEMENT OF PEDIATRIC CLINICAL HANDOVER USING VARIOUS RECOMMENDATIONS SET BY THE NCEC, HSE - A QUALITY IMROVEMENT PROJECT}

${ }^{1}$ Liqa ur Rehman*, ${ }^{2}$ Ashfaq Ahmad, ${ }^{2}$ Eilaf Nour, ${ }^{2}$ Kafil Shadani. 'Portiuncula University Hospital, Lettereknny, Ireland; ${ }^{2}$ Letterkenny University Hospital, Letterkenny, Ireland

\subsection{6/archdischild-2019-epa.522}

Aim 1) To examine if clinical handover in the Paediatric unit of Level 2 Paediatric Hospital followed the National Clinical Guidelines (NCG) set by the National Clinical Effectiveness Committee (NCEC) and then to implement these guidelines in order to optimise the process of handover 2) To improve patient safety, communication and ensure the process of handover positively impacts patient care.

\section{Method}

1. Data was collected from 22 clinical handover sessions that occurred every morning on each weekday over the period of one month, prospectively and retrospectively. Data was collected for various recommendations including numbers 2 , $3,6,7,8,9,16,17,18$ and 20. Topics included under these 
recommendations were: Compliance with ISBAR, Location, Time taken, Start time, Staff attendance, Discussion of roles and responsibilities and Record keeping. The results prompted a re-audit. NCHD's were then given education sessions regarding the recommendation's. A summary of the recommendations was also placed in the clinical handover room. A re-audit was done 3 months later before change over.

2. patients were also randomly selected and were surveyed confidentially about Diagnosis, Investigations, Treatment plans and satisfactory communication to see if the team was complying with recommendation two in the NCG. Three months later, 30 patients were surveyed again.

Results It was found that:

\begin{tabular}{lll}
\hline \multicolumn{2}{l}{ Compliance $\%$ of recommendations set out by NCEC, HSE } & \\
\hline & Pre-intervention & Post intervention \\
ISBAR & $78 \%$ & $100 \%$ \\
Time taken at CH (15-30 mins) & $64 \%$ & $100 \%$ \\
CH start time (8.55am) & $73 \%$ & $100 \%$ \\
Role and responsibilities discussed at CH & $68 \%$ & $100 \%$ \\
All patients updated at CH (new and old) & $45 \%$ & $100 \%$ \\
Record keeping of handover list & $64 \%$ & $100 \%$ \\
Patients satisfactory communication & $83 \%$ & $97 \%$ \\
\hline
\end{tabular}

Conclusion Through educational sessions and teamwork a marked improvement was seen at handover after three months. Due to the changes implemented, handover became more efficient and also benefitted patients as the quality of care improved. The next step would be to start educating NCHDs at the beginning of each changeover so that handover continues to run smoothly.

\section{P168 THE PRIMARY AIM OF THIS STUDY IS TO IDENTIFY TOP CAUSES OF PAEDIATRIC EMERGENCY (PE) ATTENDANCES AND THEIR SEASONAL DISTRIBUTION}

Anwar Ayubi*. BHRUT, Romford, UK

\subsection{6/archdischild-2019-epa.523}

Methods In this observational hospital based study electronic data was analysed focusing on medical diagnosis and seasonal distribution over a period of 5 years (Sep 2013 to August 2018). Data was collected and analysed by the two authors. Excluded from the study neonates and those attended with non-medical conditions (e.g. injuries, surgical problems).

Results 32474 eligible patients attended the PE Department during the study period. Top medical diagnoses contributed to $65 \%(\mathrm{n}=21108)$ of total attendances and included fever without focus; $(n=3949 ; 12 \%)$; tonsillitis $(n=2485 ; 7.5 \%)$; gastritis and/or gastroenteritis $(\mathrm{n}=2971$; 9\%); acute bronchiolitis $(n=2042 ; 6 \%)$; bronchial asthma $(n=1602 ; 5 \%)$; upper respiratory tract infection $(n=1122 ; 3.5 \%)$; seizure $(2443$; $7.5 \%)$; skin rash $(\mathrm{n}=1772 ; 5.5 \%)$; sickle cell disease and other causes of anaemia $(n=2845 ; 9 \%)$. Seasonal distribution of most common medical diagnoses was autumn (29\%); summer (19\%); winter (27\%) and spring (25\%).

Conclusion Our findings showed convincing evidence that majority of medical diagnoses of PE attendance can be managed by an experienced general paediatricians and paediatric nurses. Furthermore, we found there was no considerable seasonal variation among these medical causes. .

\section{P169 AUDIT OF EPILEPSY PRACTICE IN A DISTRICT GENERAL HOSPITAL}

${ }^{1}$ Sairah Akbar*, ${ }^{2}$ Rosemary Grattan, ${ }^{2}$ Lesley Nairn. ${ }^{1}$ NHS Greater Glasgow and Clyde, Glasgow, UK; ${ }^{2}$ Greater Glasgow and Clyde, Glasgow, UK

\subsection{6/archdischild-2019-epa.524}

Epilepsy is a common disorder affecting 0.5 to $1 \%$ of the paediatric population. To improve patient outcomes and ensure provision of high-quality care, NICE have detailed quality standards in their 2013 document. ${ }^{1} \mathrm{~A}$ retrospective audit was carried out in 2018 in a district general hospital to review adherence to these standards. 104 patients were identified - 33 were under the care of a tertiary specialist with the remaining 71 looked after by General Pediatricians. This subgroup was included in further analysis. 54/71 were seen by Pediatricians with expertise in epilepsy with remaining 17 by General Pediatricians with no specific interest. Almost all patients (69/71) had contact with the Epilepsy Specialist Nurse; those that did not were looked after by non-experts in this field. 8 patients were appropriately referred to tertiary care with diagnostic challenge and lack of improvement on anti-epileptic medication being the commonest reason for referral. Almost all patients had a seizure classification (69/71) with this readily available in the notes in $79 \%$ of patients and all patients were appropriately imaged.

Our audit of practice in a district general has shown the standard of care provided to paediatric Epilepsy patients conforms well to national guidelines. Areas for improvement are to ensure ESN are involved in the care of these patients and use of the International League Against Epilepsy (ILAE) classification. $^{2}$

\section{REFERENCES}

1. Epilepsy in children and young people. NICE guidelines (2013)

2. The 2017 ILAE Classification of Seizures

\section{P170 A STUDY OF IRISH TRAINEE DOCTORS PRESCRIBING KNOWLEDGE FOR THE DIFFERENT MEDICATION OPTIONS FOR ADHD}

${ }^{1,2}$ Kate Irvine*, ${ }^{3,4}$ Professor Fiona McNicholas. 'St John of Gods Hospital, Dublin, Ireland; ${ }^{2}$ Irish College of Psychiatry, Dublin, Ireland; ${ }^{3}$ Our Lady's Children's Hospital, Dublin, Ireland; ${ }^{4}$ University College Dublin, Dublin, Ireland

\subsection{6/archdischild-2019-epa.525}

Introduction Attention-deficit/hyperactivity disorder (ADHD) is a common treatable childhood mental illness, with a prevalence of $7 \%$ in under 18 year olds. Functional impairment affects all environments and includes inattention impeding memory and learning, hyperactivity resulting in increased restlessness and movement, and impulsivity leading to poor judgement and risk taking behaviour. These impairments affect children's social inclusion and ability to integrate and enjoy life, but also their ability to fulfil important educational goals. Children and adolescents with ADHD are more likely to have co-morbid medical conditions, which in turn likely impacts on 